\title{
A multicentre retrospective cohort comparison of aetiology and survival in patients with chronic hypersensitivity pneumonitis versus idiopathic pulmonary fibrosis
}

Barratt, SL : Bristol Interstitial Lung Disease Service, North Bristol NHS Trust, Bristol, UK

Adamali, HI : Bristol Interstitial Lung Disease Service, North Bristol NHS Trust, Bristol, UK

Creamer, A : Bristol Interstitial Lung Disease Service, North Bristol NHS Trust, Bristol, UK

Duckworth, A : University of Exeter Medical School, Exeter, UK

Fallon, J : Somerset Lung Centre, Musgrove Park Hospital, Taunton, United Kingdom

Gibbons, MA : Respiratory Department Royal Devon and Exeter NHS Foundation Trust Exeter, United Kingdom \& University of Exeter Medical School, Exeter, UK

Gooptu, B : Leicester Institute of Structural and Chemical Biology / NIHR Leicester BRC - Respiratory, University of Leicester, Leicester, UK \& Institute for Lung Health, Glenfield Hospital , University Hospitals of Leicester NHS Trust, Leicester UK

Nancarrow, T : University of Exeter Medical School, Exeter, UK

Pepperell, J : Somerset Lung Centre, Musgrove Park Hospital, Taunton, United Kingdom

Stone, RA : Somerset Lung Centre, Musgrove Park Hospital, Taunton, United Kingdom

Woodhead, FA : Institute for Lung Health, Glenfield Hospital , University Hospitals of Leicester NHS Trust, Leicester UK

Scotton, CJ : University of Exeter Medical School, Exeter, UK

Introduction: Hypersensitivity pneumonitis is a complex syndrome resulting from repeated inhalation and sensitisation to a variety of antigenic stimuli. The majority of cases are classified as chronic and may result in fibrotic progression which can be hard to distinguish from that of idiopathic pulmonary fibrosis (IPF). Precise aetiology and optimal management strategies are less well defined than for IPF, although clinical trials exploring the use of pirfenidone (NCT02496182) and nintedanib (NCT02999178) are in progress.

Objectives: To establish a large, UK-based, multicentre, retrospective cohort of patients with cHP to facilitate detailed investigation of patient demographics, management and prognosis.

Methods: We performed a multicentre evaluation of clinical data for patients who presented to the interstitial lung disease clinics at the North Bristol NHS Trust, University Hospitals of Leicester NHS Trust, Taunton and Somerset NHS Foundation Trust and the Royal Devon \& Exeter NHS Foundation Trust and who required multidisciplinary team evaluation between 2005 and 2018. Basic 
demographics and survival were compared using chi-square test with Yates correction (gender), Mann-Whitney U-test (age at presentation) and Kaplan-Meier Survival analysis in Graphpad Prism.

Results: In total, 397 patients with a diagnosis of cHP were identified, compared with 1068 patients with IPF. There was a significant difference in age at diagnosis between CHP and IPF (median $72.0 \mathrm{vs}$ 75.6, $\mathrm{p}<0.0001$ ), and a highly significant difference in gender distribution, with a greater proportion of women presenting with cHP than men ( 237 female vs 160 male, $p<0.001$ ), in stark contrast to cases of IPF ( 246 female vs 822 male). cHP is associated with a better prognosis than IPF ( $p<0.05)$, with a median survival of 7.1 years following diagnosis compared with 3.0 years for IPF.

Conclusions: Despite challenges in diagnosing cHP (as demonstrated by Walsh et al ${ }^{1}$ in their review of UK MDT agreement where HP has a fair kappa score of 0.29 ) and in line with published literature, our cHP cohort has a female preponderance and is associated with better survival than IPF. This cohort will provide a crucial resource for more detailed analysis of patient data (e.g. spirometry and response to therapy).

1) Walsh S.L., Wells A.U. Multicentre evaluation of multidisciplinary team meeting agreement on diagnosis in diffuse parenchymal lung disease: A case-cohort study. Lancet Respir. Med. 2016;4:557-565. doi: 10.1016/S2213-2600(16)30033-9 